\title{
BMJ Open Oral health improvement for nursing home residents through delegated remotivation and reinstruction (MundZaRR Study): study protocol of a cluster-randomised controlled trial
}

Katrin Hertrampf, ${ }^{1}$ Peter Schlattmann, ${ }^{2}$ Gabriele Meyer, ${ }^{3}$ Georg Gassmann, ${ }^{4}$ Jens Abraham, ${ }^{3}$ Volker Hammen, ${ }^{5}$ Falk Schwendicke (i) ${ }^{6}$

To cite: Hertrampf $K$, Schlattmann P, Meyer G, et al. Oral health improvement for nursing home residents through delegated remotivation and reinstruction (MundZaRR Study): study protocol of a cluster-randomised controlled trial. BMJ Open 2020;10:e035999. doi:10.1136/ bmjopen-2019-035999

- Prepublication history for this paper is available online. To view these files, please visit the journal online (http://dx.doi. org/10.1136/bmjopen-2019035999).

Received 25 November 2019 Revised 05 June 2020 Accepted 07 August 2020

Check for updates

(C) Author(s) (or their employer(s)) 2020. Re-use permitted under CC BY-NC. No commercial re-use. See rights and permissions. Published by BMJ.

For numbered affiliations see end of article.

Correspondence to

Dr Falk Schwendicke;

falk.schwendicke@charite.de

\section{ABSTRACT}

Introduction Oral health and oral health-related quality of life $(\mathrm{OHrQL})$ of residents in German long-term residential care (LRC) are poor. We will develop an evidence-based catalogue of interventions ('Oral Health Toolbox') and provide care-accompanying reinstruction and remotivation of nursing staff by dental assistants (DA). We hypothesise that such intervention will significantly improve $\mathrm{OHrQL}$, daily oral hygiene/care behaviour and is cost-effective.

Methods and analysis A scoping review will be used to identify possible intervention components. Mixed methods will be used to identify barriers and enablers of oral hygiene and care in German LRC. The result will be the 'Oral Health Toolbox', a two-phased instrument supporting both initial intervention allocation to improve oral health/ hygiene and reinstruction/remotivation. A two-arm clustered, randomised controlled trial (ratio of 1:1 via block randomisation) will be performed in LRC in RhinelandPalatinate, Germany. Each nursing home represents a cluster. Based on a feasibility study, considering clustering and possible attrition, we aim at recruiting 618 residents in 18 clusters. In the intervention group, dentists will assign one or more intervention component from the box (phase 1). During follow-up, nursing staff will be reinstructed and remotivated by DA, who use the box to decide how to maintain the intervention (phase 2). In the control group residents will receive care as usual. The primary outcome, OHrQL, will be measured using the General Oral Health Assessment Index. Secondary outcomes include pain condition, general health-related quality of life, caries increment, oral/prosthetic hygiene and gingival status, incidence of dental emergencies and hospitalisations, and cost-utility/effectiveness. The endpoints will be measured at baseline and after 12 months. For our primary outcome, a mixed-linear model will be used within an intention-totreat analysis. A process evaluation using mixed methods will be conducted alongside the trial.

Ethics and dissemination Ethical approval by the University of Kiel was granted (D480/18).

Trial registration number NCT04140929.
Strengths and limitations of this study

- This is the first cluster randomised large-scale study testing an oral health-focused intervention in German nursing homes.

- The complex intervention is innovative, building on the toolbox and interprofessional care.

- Aspects of application and implementation in daily care are considered throughout the study.

- The study will not be fully blinded, and we expect early termination in this specific group.

- Generalisability will be restricted to German nursing homes.

\section{INTRODUCTION}

Currently, there are approximately 800000 people living in nursing homes in Germany. Of these, an estimated $40 \%-70 \%$ show considerably limited daily skills, mainly but not exclusively due to dementia. ${ }^{1-3}$ These limited daily skills, including lacking mobility, affect access to oral hygiene and care, and are risk factors for inadequate oral hygiene. ${ }^{4}$ As more and more older people have more and more teeth of their own, most of which are restored with extensive removable restorations or fixed dental prostheses, the demands for oral hygiene and care increase. ${ }^{35}$

Results of the Fifth German Oral Health Study (DMS V) highlight that the oral hygiene and care currently provided in German longterm residential care (LRC) is inadequate. The majority of the investigated LRC residents showed a high number of untreated carious lesions, periodontitis and poorly fitting full or partial removable prostheses, ${ }^{5}$ resulting not only in low oral health-related quality of life (OHrQL), but also a high need for dental emergency treatment and comorbidities (eg, pneumonia, hyperglycaemia, 
malnutrition). ${ }^{6} 7$ A range of approaches have been suggested to improve the oral hygiene and health of LRC residents. $^{8-10}$

The German Federal Dentists Association and the National Association of Statutory Health Insurance Dentists (KZBV) developed the national concept entitled 'Oral health despite handicap and high age' as one response to this situation in 2014, with dentists now being able to contractually cooperate with LRC. Within this contract, a biannual (6 monthly) examination to determine dental, oral and maxillofacial diseases is included. Dentists use a standard form to record any findings and provide caregivers with instructions towards oral hygiene and care. Dentists can, in theory, also provide care within the LRC, while this is not common at the moment. ${ }^{11}$ By the end of 2016, about $6 \%$ of dentists signed such a cooperation agreement. ${ }^{12} 13$ Recent further measures include the option to provide individual preventive measures like oral hygiene and nutrition advice as well as fluoridation to the nursing home residents (Richtlinie nach §22a SGB V(neu)). However, dentists do not receive any standardised training for preparing them for working within LRCs, and there is also no quality assurance in place. Overall, it remains uncertain which interventions dentists chose to address LRC residents' needs, if they regularly provide the available preventive measures, and if the usage of the standard form is sufficient to truly alter the daily oral care in LRC. Overall, it is unclear such care is effective, implementable and sustainable.

A feasibility mixed-methods study, aiming to assess if interprofessional maintenance interventions are useful to sustainably improve oral hygiene and care, was carried out in the State of Rhineland-Palatinate, Germany. Forty nursing home residents were assessed and cared jointly for by nursing home staff and dental assistants (DAs) for 6 months. The DAs trained, reinstructed and remotivated nurses to provide oral hygiene and care measures in daily care. Although the study lacked theoretical underpinning and was not powered to demonstrate any effectiveness with confidence, it revealed some important hints in OHrQL (measured via the German version of the Oral Health Impact Profile (OHIP-G14) $)^{14}$ that effectiveness may be given. The qualitative analyses demonstrated the importance of interprofessional cooperation for process quality (unpublished data).

We therefore plan to conduct a cluster-randomised controlled trial (cRCT), addressing both the issue of choosing the most effective interventions for improving oral hygiene and care, and how to maintain this effectiveness in the long-term via intermittent interprofessional cooperation. This cRCT takes into account the legal delegation framework covered by the German Law of Dentistry (Zahnheilkundegesetz). This includes that a DA cannot independently decide and carry out measures, for example, for motivation and instruction of oral hygiene for LRC residents, as this would correspond to substitution and is excluded by this law. Hence, all necessary measures need to be consciously and actively delegated to the DA by the dentist.

We will use an 'Oral Health Toolbox' which allows the dentist to choose the most effective intervention for each individual LRC resident, and the DA to identify areas where maintenance may be compromised and address them during reinstruction and remotivation of the nursing staff during daily care. Our research questions are as follows:

1. Can an individually tailored, evidence-informed intervention, with care-accompanying reinstruction and remotivation of nursing staff by $\mathrm{DA}$, improve $\mathrm{OHrQL}$ and oral health?

2. Does such reinstruction and remotivation facilitate a change in the behaviour of nursing staff towards oral hygiene and oral care, and can it be successfully implemented in daily care?

3. What is the cost-utility/effectiveness ratio of such an intervention?

\section{METHODS AND ANALYSIS Overview and setting}

We are planning an intervention that draws on defined, individualised components from an 'Oral Health Toolbox' (which we will develop) (1) to decide which interventions to apply to each individual LRC resident and (2) how to maintain them. The latter will be supported via reinstruction and remotivation of nursing staff by DA during regular care. The testing of the effectiveness of this complex intervention is planned in a two-arm parallel group cRCT, with the control group being 'Standard of Care'. This is a study which is carried out over 12 months, with initial examinations (T0) and re-examinations after 6 months (T1) and 12 months (T2). The original launch of the cRCT was scheduled for 1 April 2020. Recruitment was stopped at the beginning of March due to the COVID-19 pandemic. Given current dynamics, we expect the cRCT to start on 1 April 2021. The end of the the cRCT would therefore be March 2022. The contractual cooperation between dentists and nursing homes obliges the dentist to carry out 6 monthly visits. We have taken this standard into account when determining our measurement dates. Given the duration of stay of individuals in LRC in Germany, longer trial periods may come with unacceptably high attrition. The setting will be nursing homes of the Marienhaus holding, located in RhinelandPalatinate, Germany. The Standard Protocol Items: Recommendations for Interventional Trials ${ }^{15}$ checklist is provided as online supplemental material 1.

\section{Development of the Oral Health Toolbox}

A scoping review ${ }^{16}{ }^{17}$ of established intervention components will be conducted. We are including comparative interventional studies, that is, randomised or nonrandomised trials, investigating interventions to improve oral care, oral hygiene and oral health in LRC on seniors (aged 65 years or above). No further restrictions as to study 
type, participants, intervention focus or method, delivery or outcome measures will be applied; for example, interventions could target prevention of oral conditions, but also their treatment, by dentists, DAs or nursing staff. We will search Medline (via PubMed), Embase via Ovid and CINAHL. Search strategies will be adapted for each database; the following search strategy will be used for PubMed: (elderly) OR seniors) OR elder) AND (dental) OR oral) OR teeth) OR tooth) OR lip) OR tongue) AND (care home) OR care facility) OR nursing)) AND (trial) OR randomized) OR randomised) OR random) OR randomly) OR vivo) OR clinical). No time limitation for the publication date will be set. Our search will be limited to the following languages: English, German, Swedish, Danish, Norwegian and Spanish. We will extract data on interventions (target group, provision, design, maintenance) and outcomes. We will assess the effectiveness of different interventions or their components, using qualitative and/or quantitative syntheses, and classify interventions as to their focus during the care process (diagnosis/ screening of oral conditions; daily maintenance of oral health; provision of dental treatment). Interventions or their components will be assigned to the domains capability, opportunity and motivation of the Behaviour Change Wheel (BCW) ${ }^{18}$ The BCW is used to understand causal factors that influence behaviour. The result of this synthesis will be a systematised catalogue of intervention components, informing on (1) their measured effectiveness within the studies at hand and (2) the behaviour domain each intervention is supposed to work through; the latter will allow to reverse-engineer the Oral Health Toolbox based on the identified most-pressing barriers and enablers to provide oral hygiene and care in German LRC settings.

Next, mixed-method studies will be performed to better understand which interventions or their components are likely to be effective, implementable and sustainable within German healthcare. We plan qualitative studies using semi-structured focus group discussions or single interviews to identify barriers and enablers of oral hygiene and care in German LRC, and to evaluate and adapt the collected intervention components according to the specific needs of German care. The sampling for the qualitative studies will be purposively or according to the snowball principle. We plan with an expected number of cases of approximately 15 nursing staff, 5 nursing home residents and 5 other stakeholders, for example, nursing home directors, family members of residents and dentists servicing in LRC. Nursing staff will be interviewed separately from home directors. Audio recordings and corresponding transcripts will be assessed using thematic analyses along the Theoretical Domains Framework (TDF). The TDF is an instrument for systematising behavioural aspects in implementation research and contains 14 domains. ${ }^{19}$ Identified barriers and enablers for oral hygiene and care will be systematised along the TDF and linked to the BCW domains (figure 1). Hence, we will be able to reverse-engineer the toolbox, building on the identified daily problems in care and assigning effective intervention to them, categorised according the location within the care process. Moreover, we will use the established framework to develop a decision-aid for DA to identify areas where the maintenance has been compromised during follow-up, and to target these areas specifically during reinstruction and remotivation. The result, the 'Oral Health Toolbox', will contain two parts (one on the initial interventions a dentist can assign, tailored to each patient, and one a DA will use during follow-up to identify areas which will be addressed during reinstruction and remotivation), delivered on a two-page leaflet. The box will not be accompanied by any specific care products, as its focus is to provide the dentists with a set of possible interventions and the DA with an algorithm as to how to reassess oral hygiene and care prior to reinstruction/remotivation. The application of the box will be trained (see below), but will allow flexibility to adapt to individual settings and needs. The box allows the dentists sufficient freedom to choose specific measures not only based on their assessment, but also expertise and the patient's needs. During reinstruction and remotivation, the box will be more specific and guide the DA in a more formalised way. Both the first and second application of the box will be documented, as will be the care process in daily life.

\section{Participants, sample size and recruitment}

The target population are LRC residents. We will only include facilities without an existing dental cooperation agreement. Eligible are residents with a degree of care dependency of 3-5 (moderate to severe) as rated by an expert rater of the German statutory health insurance. Note that German LRCs do not provide hospice care, which means individuals with such care will not be captured. Exclusion criteria are a lack of consent (of the home management and/or the residents or their proxies or legal guardians) and residents in respite care. The consent forms are provided as online supplemental materials 2,3. The different number of residents included in each nursing home is taken into account in the block randomisation. Details on the nursing homes and their staff, as well as of each resident will be collected in a standardised manner.

The results of the feasibility study are used to calculate the number of cases. Initially, an average OHIP-G14 of 14.0 was measured. This was reduced by the intervention to 6.5 (delta=7.5, $\mathrm{SD}=14.0)$. We assume to observe at least this effect size with the given variance within our study using the questionnaire General (formerly Geriatric) Oral Health Assessment Index (GOHAI) (see below) to measure the OHrQL. Under these assumptions and using an unpaired t-test, a total of 170 subjects ( 85 per group) are required at a significance level of $5 \%$ (two-sided) and a power of $80 \%$. The cluster randomisation additionally increases the required number of subjects by a factor of 2.75 , with 34 subjects per institution and an intracluster correlation of $0.05 .^{20}$ The target sample size per arm is 


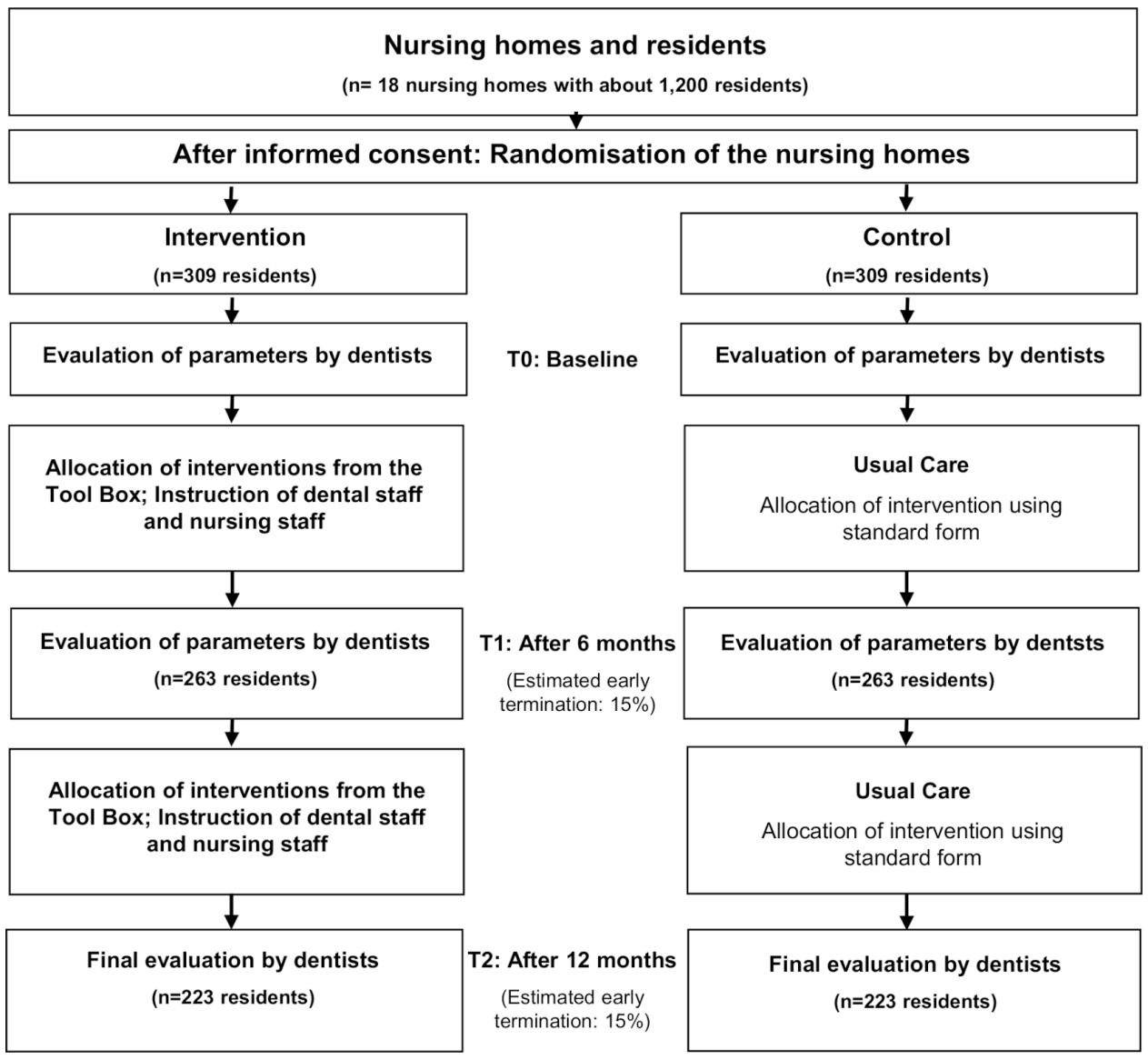

Figure 1 Study flowchart.

therefore 238 subjects. Considering the proportion of residents who terminate the study early due to death or severe morbidity (30\%), a total of 618 residents (in a total of approximately 18 facilities) are required. The Marienhaus holding has a total of 28 facilities in RhinelandPalatinate and supports recruitment. The planned flow of the participating institutions and participants through the study is shown in figure 1.

\section{Allocation and blinding}

A cluster is defined as an LRC. The LRC will be randomised either to the intervention group or the control group at a ratio of 1:1. Randomisation will be performed externally in the Institute of Medical Statistics at the University Hospital Jena by means of block randomisation with variable block length. The biometrician will be blinded. Neither the dentists using the box during the initial assessments, nor DAs nor nursing staff nor participants can be blinded with regard to intervention assignment.

\section{Intervention and control group}

In the intervention group, a range of clinical parameters (see below) are recorded and the dentists prepare an individual oral hygiene and care recommendation, in addition to a standardised form (see control group), based on the Oral Health Toolbox. The box is also used for instructing the DA and the nursing staff for each patient individually. It is then determined when and how the DA re-evaluates the oral hygiene and care process and reinstructs and remotivates the nursing staff. If the examination reveals a need for dental treatment (possibly requiring referral and transport), this will be communicated to the nursing staff via the standard form. Note that this part, for example, provision of dental treatment, is identical in both study arms. The DAs will further receive a training in communication, enabling them in reinstruction and remotivation. Since the DAs are continuously in the nursing homes, the reinstruction will also cover new nursing staff. Note, though, that we assume not every staff member to receive all reinstruction and remotivation given shift duties.

In the control group, the residents receive care as usual. This includes the recording of the same parameters are recorded as in intervention group. A standard form is filled out and handed over to the nursing staff. As in the case of intervention group, the nursing staff is informed in the event of a need for treatment. No further measures are applied.

In both the intervention and control group, we will record what was provided during the initial examination and advice session, during reinstruction and remotivation, and during daily oral care; the latter will be done via routine forms (which will be minimally adapted for the intervention group). Furthermore, the study is accompanied by a comprehensive process evaluation, and after 
completion of the intervention, by a routine data and health economic analysis. We do not expect any adverse effects from the intervention.

\section{Staff}

Twelve dentists and four DAs for both intervention and control group are recruited via existing dentists/practice networks and in cooperation with the Dental Association of Rhineland-Palatinate. All teams will be calibrated by the project management, co-management and coordinator in the facility. The calibration for the collection of outcomes and further parameters (see below) will be carried out according to the established procedure for field dentists of the DMS. ${ }^{21}$ In addition, the digital data input system and data transfer will be introduced. After 6 months, the recalibration for the dentists will take place.

\section{Outcomes}

Our primary endpoint, the OHrQL, will be assessed by the GOHAI. The GOHAI is an established instrument for measuring OHrQL in older people. It comprises twelve items in four domains (functional limitations, pain/ discomfort, psychological aspects, behavioural aspects), which are recorded ordinally scaled from 0 (never true) to 5 (always true). The GOHAI shows high discriminatory validity, construct validity and criterion validity. Compared with other OHrQL instruments widely used in dentistry (eg, OHIP-14), the GOHAI is able to reflect functional limitations, self-assessed oral health and satisfaction with one's own oral health status in an elderly population. ${ }^{22}$ The GOHAI has been validated with regard to validity, reliability and discrimination in German-speaking countries. ${ }^{23}{ }^{24}$ Note that in our specific target population, the application of the GOHAI may be problematic in some instances. However, the survey can be administered in multiple ways, for example, by self-fillout, by reading it to the participant, who may answer verbally or by pointing to the response, as well as by relying on staff or family members to assist in the survey. Note that if the survey is provided purely by staff or family, this will be noted and considered in a sensitivity analysis. Also note that we collect clinical, objectified data as well as data on pain (see below) to triangulate them with the GOHAI.

Secondary endpoints and survey instruments will be:

- Pain condition, measurement of actual pain by scale (1-10), 1-least pain; 10 -most pain.

- Health-related quality of life, measured using European Group Quality of life 5 Dimensions-5 Levels. ${ }^{25}$

- Caries experience/tooth loss, measured with DecayedMissing-Filled Teeth (DMFT) and root caries lesions.

- Oral hygiene status, measured using Geriatric Debris Index. ${ }^{26}$

- Gingival inflammation, measured using Periodontal Screening Index. ${ }^{27}$

- Prosthetic hygiene, measured using Denture Hygiene Index. $^{28}$

- Health economic analysis, measured using Incidence of Dental-Associated Emergencies and
Hospitalisations and Nursing, dental and inpatient treatment costs and indirect costs (see chapter health economic analysis).

The endpoints will be measured at T0 (baseline) and $\mathrm{T} 1$ and T2 (6/12 months). The analytical metric will be the change from $\mathrm{T} 0$ to $\mathrm{T} 2$.

\section{Data management}

Data management is carried out by a qualified data manager at the University Hospital Jena. All documentation is digital. Measures carried out are documented in a corresponding data mask. The required data input masks and software are programmed or adapted according to the DMS V model. ${ }^{21}$ After collection, the data is pseudonymised and transferred to the data centre via a transfer mode established under data protection law, where it is checked and cleansed. All data protection regulations are applied.

\section{Clinical data analysis}

The primary endpoint will be evaluated using a mixed linear model with the covariates treatment, time, baseline GOHAI values, subject age and gender, degree of care dependency and a random effect for the homes and residents. The random effects introduce possible cluster effects and the correlation within residents and possible unobserved variables. This analysis is performed primarily for the intention-to-treat (ITT) population. In the ITT population, at least one intervention and one follow-up examination have taken place. The evaluation for the per-protocol population takes place as a secondary analysis. Secondary endpoints are assessed descriptively and evaluated exploratively, with generalised mixed models according to scale. Missing values are replaced in a sensitivity analysis via multiple imputation.

\section{Health economic analysis}

The primary health economic evaluation will be a costutility analysis. The costs will be collected from the perspective of the health insurance party payer. The OHrQL (GOHAI) will be used as the utility value. In addition, cost-effectiveness analyses are carried out, in which the effectiveness measure will be clinical parameters (DMFT). The time horizon of the analyses will be 12 months. In addition, model-based lifetime analyses will be used; these will also serve to analytically record parameter and structural uncertainty or heterogeneity (see below). No discounting is planned within the 12-month analysis horizon; for extrapolated (model-based) analyses, costs and utility or effectiveness are discounted at $3 \%$ per annum in order to account for the positive time preference and opportunity costs of capital. ${ }^{29}$

To record costs, both quantity and price units are recorded (marginal analysis). The following cost-related parameter will be recorded: (1) nursing staff hours spent on daily oral care; (2) costs for oral health-related equipment/material used; (3) dentist hours spent for examination, intervention assignment and either filling the 
standard form or instruction of the DA and the nurse; (4) DA hours spent during initial examination and reinstruction/remotivation; (5) costs for dental services itemised using fee items of the statutory insurance, provided from routine claims data collected by the local KZBV within a data exchange agreement; (6) costs for other medical treatment which can be clearly related with oral/dental health (we assume this to be the exception), collected at each re-evaluation via record audit. Note that, as described, dental and further medical treatment itself is not provided as part of this trial and will be provided as is standard in each nursing home, likely ranging from care onsite to transporting individuals to practices. Nevertheless, we are interested to learn if, for example, our intervention reduces the need for dental treatment and hence costs, which is why we collect these data as described. Consumption costs or structural or administrative costs are recorded on a flat-rate basis. Subgroup analysis will be performed to discriminate trial-related costs from those resulting from the intervention(s). Since any opportunity costs of the residents are difficult to record and are already considered on the effects side, they will not be included separately here.

Incremental cost-utility ratios are used for comparative analyses. We will carry out univariate and bivariate sensitivity analyses to assess the influence of parameter uncertainty (utility value, costs) and heterogeneity (eg, stratification according to degree of care, age, gender of residents). In addition, model-based analyses (Markov models) are carried out; this also allows the influence of structural (model) uncertainty to be assessed. Using Monte Carlo analyses, both extrapolation and probabilistic analysis to describe 'joint uncertainty' ('joint parameter uncertainty') will then be performed. Here cost-use-value-acceptability curves are used for analysis. $^{30} 31$

\section{Process evaluation}

The process evaluation aims at making changes in the course of the complex intervention understandable. The mixed-methods study is based on established guidelines. ${ }^{32} 33$ The aim is to develop a theory-based model of changes (Theory of Change) in order to map context factors (eg, framework conditions, organisational structures), implementation (eg, type of introduction, extent, time period) and the mechanisms of action (eg, also interactions) of the intervention. ${ }^{34}$ The data collection of the process evaluation is shown in table 1 .

If no validated tools are available, survey and interview measures will be developed and pre-tested. An evaluation

Table 1 Data collection during process evaluation

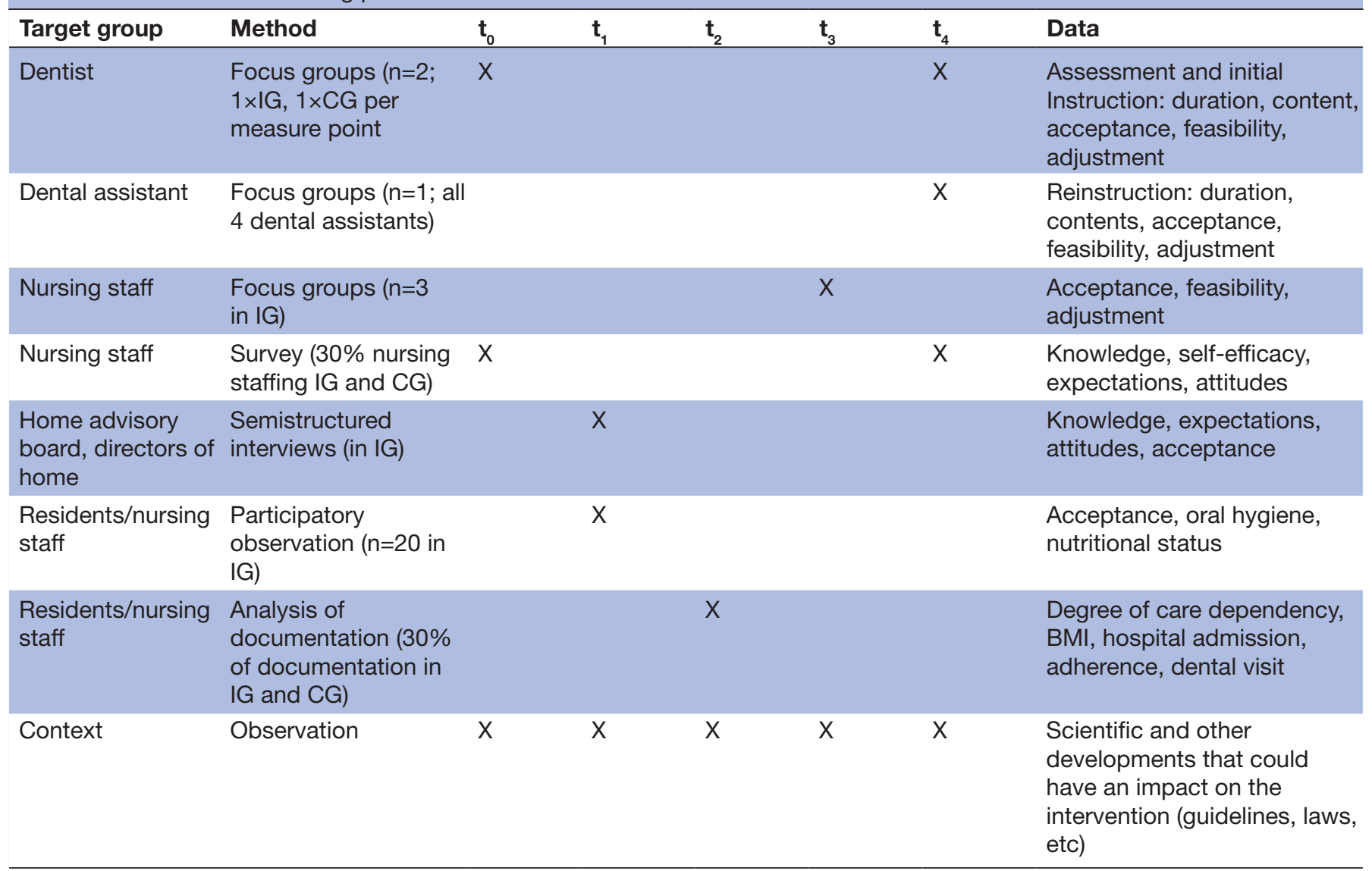

Measurement points $t_{0}=$ baseline; $t_{1}=3$ rd month; $t_{2}=6$ th month; $t_{3}=9$ th month; $t_{4}=12$ th month .

$\mathrm{BMI}$, body mass index; CG, control group; IG, intervention group. 
matrix will be developed and pre-tested for qualitative analyses. The focus groups and the individual interviews will be tape-recorded. The transcripts will be evaluated using qualitative content analysis. ${ }^{35}$ The eight dentists and the DA will be interviewed in separate focus groups at the beginning and at the end of the intervention. For the nursing home staff, three focus groups are planned in order to assess acceptability, feasibility and adjustments. In addition, $30 \%$ of the staff will be surveyed at the beginning and at the end of the intervention on knowledge, self-efficacy, expectations and attitudes. The knowledge, expectations and acceptance of the residents regarding the intervention will be assessed as well. In order not to have to rely solely on self-assessments, the acceptance and behavioural changes (eg, oral hygiene, nutrition) of 20 residents in the intervention group will be recorded by means of participatory observation. In addition, a document analysis will be performed based on $30 \%$ of the documentation in the intervention and control group in order to determine objective characteristics such as degree of care dependency, hospital admissions, adherence or dental visits.

\section{Ethics and dissemination}

The study is conducted in accordance with the Declaration of Helsinki, ${ }^{36}$ the ICH Guideline on Good Clinical Practice $^{37}$ and the Federal Data Protection Act (BDSG, SGB V/X for the processing of personal patient data). The study protocol was submitted to the Ethics Commission of the Medical Faculty of the University of Kiel for ethical review and for an initial vote (D480/18). A regional vote was obtained from the Rhineland-Palatinate Medical Association. All participants or their proxies or legal guardians will be informed in writing and verbally and a written informed declaration of consent for the study and the data access via the regional Statutory Health Insurance Dentists will be obtained. Any deviations from the protocol will be discussed within the Advisory Board, noted in the register and indicated accordingly in any subsequent publications. All study results will be reported in accordance to this study protocol and to the Consolidated Standards of Reporting Trials statement extended to cluster-randomised trials. ${ }^{38}$

\section{Patient and public involvement}

Neither patients nor the public were involved in the design of the study, including conceiving the research questions and deciding the outcomes. However, our study will assess the subjective effects on the residents in nursing homes, as described.

\footnotetext{
Author affiliations

${ }^{1}$ Clinic of Oral and Maxillofacial Surgery, University Hospital Schleswig-Holstein, Kiel, Germany

${ }^{2}$ Medical Statistics, Computer and DataSciences, Friedrich-Schiller-Universitat Jena, Jena, Germany

${ }^{3}$ Institute for Health Care and Nursing Studies, University Halle, Halle/Saale, Germany

${ }^{4}$ praxisHochschule pHfG Trägergesellschaft, praxisHochschule University of Applied Sciences, Cologne, Germany
}

${ }^{5}$ praxisHochschule University of Applied Sciences, praxisHochschule University of Applied Sciences, Cologne, Germany

${ }^{6}$ Zahnerhaltung, Charite Universitatsmediz in Berlin Campus Benjamin Franklin, Berlin, Germany

Contributors $\mathrm{KH}$ and FS conceived and designed the study, planned data recording and analysis, and wrote the manuscript. PS, GM, GG, JA, and VH planned data recording and analysis, and revised the manuscript. All authors have agreed to the final version of this manuscript and accepted responsibility for it.

Funding This study is funded by the Innovationsfond des Gemeinsamen Bundesausschusses (01VSF18021). Open Access publication is supported by the DFG OA Fund of the Charité-Universitätsmedizin Berlin.

Competing interests None declared.

Patient and public involvement Patients and/or the public were not involved in the design, or conduct, or reporting, or dissemination plans of this research.

Patient consent for publication Not required.

Provenance and peer review Not commissioned; externally peer reviewed.

Open access This is an open access article distributed in accordance with the Creative Commons Attribution Non Commercial (CC BY-NC 4.0) license, which permits others to distribute, remix, adapt, build upon this work non-commercially, and license their derivative works on different terms, provided the original work is properly cited, appropriate credit is given, any changes made indicated, and the use is non-commercial. See: http://creativecommons.org/licenses/by-nc/4.0/.

\section{ORCID iD}

Falk Schwendicke http://orcid.org/0000-0003-1223-1669

\section{REFERENCES}

1 Abraham J, Kupfer R, Behncke A, et al. Implementation of a multicomponent intervention to prevent physical restraints in nursing homes (imprint): a pragmatic cluster randomized controlled trial. Int $J$ Nurs Stud 2019;96:27-34.

2 Statistisches Bundesamt. Pflegestatistik 2015. Wiesbaden: destatis, 2017.

3 deStatis. Pflegestatistik 2013, 2015.

4 Zimmerman S, Austin S, Cohen L, et al. Readily identifiable risk factors of nursing home residents' oral hygiene: dementia, hospice, and length of stay. J Am Geriatr Soc 2017;65:2516-21.

5 Nitschke I, Micheelis W. Krankheits- und Versorgungsprävalenzen bei Älteren Senioren mit Pflegebedarf. In: Jordan R, Micheelis W, eds. Fünfte Deutsche Mundgesundheitsstudie (DMS V). Köln: Deutscher Zahnärzte Verlag DÄV, 2016: 557-78.

6 Van der Maarel-Wierink CD, Vanobbergen JNO, Bronkhorst EM, et al. Oral health care and aspiration pneumonia in frail older people: a systematic literature review. Gerodontology 2013;30:3-9.

7 Chapple ILC, Bouchard P, Cagetti MG, et al. Interaction of lifestyle, behaviour or systemic diseases with dental caries and periodontal diseases: consensus report of group 2 of the joint EFP/ORCA workshop on the boundaries between caries and periodontal diseases. J Clin Periodontol 2017;44 Suppl 18:S39-51.

8 Weintraub JA, Zimmerman S, Ward K, et al. Improving nursing home residents' oral hygiene: results of a cluster randomized intervention trial. J Am Med Dir Assoc 2018;19:1086-91.

9 Rozas NS, Sadowsky JM, Jeter CB. Strategies to improve dental health in elderly patients with cognitive impairment: a systematic review. J Am Dent Assoc 2017;148:236-45.

10 Chalmers J, Pearson A. Oral hygiene care for residents with dementia: a literature review. J Adv Nurs 2005;52:410-9.

11 Rädel M, Bohm S, Priess H-W, et al. Zahnreport 2018.Schriftenreihe Zur Gesundheitsanalyse. Barmer. Siegen: Müller Verlagsservice, 2018.

12 Bundesvereingiung K. Agenda Mundgesundheit. Köln: Kassenzahnärztliche Bundesvereingiung (KZBV), 2017.

13 KZBV. 4,331 Dental cooperation agreement for nursing homes [4.331 Kooperationsverträge für Pflegeheime]. Zm 2019;109.

14 John MT, Patrick DL, Slade GD. The German version of the Oral Health Impact Profile--translation and psychometric properties. Eur J Oral Sci 2002;110:425-33.

15 Chan A-W, Tetzlaff JM, Gøtzsche PC, et al. Spirit 2013 explanation and elaboration: guidance for protocols of clinical trials. BMJ 2013;346:e7586. 
16 Colquhoun HL, Levac D, O'Brien KK, et al. Scoping reviews: time for clarity in definition, methods, and reporting. J Clin Epidemiol 2014;67:1291-4.

17 Levac D, Colquhoun H, O'Brien KK. Scoping studies: advancing the methodology. Implement Sci 2010;5:69.

18 Michie S, van Stralen MM, West R. The behaviour change wheel: a new method for characterising and designing behaviour change interventions. Implement Sci 2011;6:42.

19 Atkins L, Francis J, Islam R, et al. A guide to using the theoretical domains framework of behaviour change to investigate implementation problems. Implement Sci 2017;12:77.

20 Richter C, Berg A, Fleischer S, et al. Effect of person-centred care on antipsychotic drug use in nursing homes (EPCentCare): study protocol for a cluster-randomised controlled trial. Implementation Sci 2015;10:1-10.

21 Jordan RA, Bodechtel C, Hertrampf K, et al. The Fifth German Oral Health Study (Fünfte Deutsche Mundgesundheitsstudie, DMS V) rationale, design, and methods. BMC Oral Health 2014;14:161.

22 Locker D, Matear D, Stephens M, et al. Comparison of the GOHAl and OHIP-14 as measures of the oral health-related quality of life of the elderly. Community Dent Oral Epidemiol 2001;29:373-81.

23 Hassel AJ, Rolko C, Koke U, et al. A German version of the GOHAI. Community Dent Oral Epidemiol 2008;36:34-42.

24 Hassel AJ, Steuker B, Rolko C, et al. Oral health-related quality of life of elderly Germans--comparison of GOHAl and OHIP-14. Community Dent Health 2010;27:242-7.

25 Ludwig K, Graf von der Schulenburg J-M, Greiner W. German value set for the EQ-5D-5L. Pharmacoeconomics 2018;36:663-74.

26 Greene JC, Vermillion JR. The simplified oral hygiene index. J Am Dent Assoc 1964;68:7-13.

27 Meyle J, Jepsen S. Der Parodontale Screening-Index (PSI). . Parodontologie, 2000: 11. 17-21.

28 Wefers K. Der Denture Hygiene Index. . Dentalforum, 1999: 9. 13-14.
29 IQWiG. Appraisal of recommendations by the scientific board of IQWiG regarding 'Methods to assess cost-effectiveness in German Public Health Insurance' [Würdigung der Empfehlung des Wissenschaftlichen Beirats des IQWiG zur Methodik für die Bewertung von Verhältnissen zwischen Nutzen und Kosten im System der deutschen gesetzlichen Krankenversicherung'], 2009. Available: https://www.iqwig.de/download/09-03-18_Wuerdigung der_Empfehlung_des_Wissenschaftlichen_Beirats.pdf

30 Drummond MF, Sculpher MJ, Claxton K, et al. Methods for economic evaluation of health care programmes. Oxford University Press, 2005.

31 Drummond M, Stoddart G, Labelle R, et al. Health economics: an introduction for clinicians. Ann Intern Med 1987;107:88-92.

32 Moore GF, Audrey S, Barker M, et al. Process evaluation of complex interventions: medical Research Council guidance. BMJ 2015;350:h1258.

33 Grant A, Treweek S, Dreischulte T, et al. Process evaluations for cluster-randomised trials of complex interventions: a proposed framework for design and reporting. Trials 2013;14:15.

34 De Silva MJ, Breuer E, Lee L, et al. Theory of change: a theory-driven approach to enhance the medical Research Council's framework for complex interventions. Trials 2014;15:267-89.

35 Mayring P. Qualitative Inhaltsanalyse: Grundlagen und Techniken. Weinheim: Beltz, 2015

36 World Medical Association. World Medical Association Declaration of Helsinki: ethical principles for medical research involving human subjects. JAMA 2013;310:2191-4.

37 Harmonisation ICo. Guideline for Good Clinical Practice E6(R1), in Internation Conference on Harmonisation of Technical Requirements for Registration of Pharmaceuticals for Human Use, 1996.

38 Campbell MK, Piaggio G, Elbourne DR, et al. Consort 2010 statement: extension to cluster randomised trials. BMJ 2012;345:e5661. 